\title{
Statistical Foundation of Empirical Isotherms
}

\author{
F. Brouers \\ Department of Chemical Engineering, Liege University, Liege, Belgium \\ Email: fbrouers@ulg.ac.be
}

Received 30 July 2014; revised 2 September 2014; accepted 12 September 2014

Copyright (C) 2014 by author and Scientific Research Publishing Inc.

This work is licensed under the Creative Commons Attribution International License (CC BY). http://creativecommons.org/licenses/by/4.0/

(c) () Open Access

\begin{abstract}
We show that most of the empirical or semi-empirical isotherms proposed to extend the Langmuir formula to sorption (adsorption, chimisorption and biosorption) on heterogeneous surfaces in the gaseous and liquid phase belong to the family and subfamily of the Burr $_{X I}$ cumulative distribution functions. As a consequence they obey relatively simple differential equations which describe birth and death phenomena resulting from mesoscopic and microscopic physicochemical processes. Using the probability theory, it is thus possible to give a physical meaning to their empirical coefficients, to calculate well defined quantities and to compare the results obtained from different isotherms. Another interesting consequence of this finding is that it is possible to relate the shape of the isotherm to the distribution of sorption energies which we have calculated for each isotherm. In particular, we show that the energy distribution corresponding to the BrouersSotolongo $(B S)$ isotherm [1] is the Gumbel extreme value distribution. We propose a generalized $G B S$ isotherm, calculate its relevant statistical properties and recover all the previous results by giving well defined values to its coefficients. Finally we show that the Langmuir, the Hill-Sips, the $B S$ and $G B S$ isotherms satisfy the maximum Bolzmann-Shannon entropy principle and therefore should be favoured.
\end{abstract}

\section{Keywords}

Adsorption Isotherms, Burr Functions, Adsorption Energy Distribution, Maximum Entropy

\section{Introduction}

Every year hundreds or more papers are devoted to the analysis of sorption (physical adsorption, chemi- and bio-sorption) of gas or solutions on a variety of substrates [2]. Among them, a great number are concerned with the decontamination of air, water and soil. One of the typical procedures is a comparison of the data with empirical isotherm formulas which in the course of time have been proposed by scientists working in the field to 
generalize the original Langmuir isotherm to heterogeneous surfaces and to sorption in solutions. Most of these formulas are empirical and bring little information on the physicochemical processes responsible for the particular shape of the isotherm curves. The evolution of the empirical parameters with external factors is recorded but there are no precise correlations between the variations of the parameters belonging to different isotherms. It appears that some order should be introduced in that field in order to propose a more rigorous classification of the sorbent-sorbate couples.

In this paper which is a contribution to that effort, we want to emphasize that since some of these isotherms appear to be genuine cumulative probability distributions, they should be favoured, formulated in the language of the theory of probability and might bring more quantitative and more structured information making advantage of their mathematical properties. The probability theory of complex systems has made considerable progress these last years and one can expect that its introduction in the field of sorption could be of great help.

\section{Sorption on Heterogeneous Surfaces}

A few years ago we published a paper [1] actualizing the efforts initiated by Langmuir, Zeldowitsch and followers eighty years ago to incorporate in the classical Langmuir adsorption isotherm theory, the heterogeneous nature of the substrate, the $\mathrm{N}$-body interactions and the nonequilibrium state of the sorbate. One important conclusion of this study was that the most important ingredient playing a role in designing the shape of the isotherm is the sorption energy distribution which itself is a reflection of the disordered and complex nature of the phenomenon. In our work, we insisted on the fact that it would be useful to rewrite the theory in the framework of the theory of probability. Moreover we reminded that it is an asymmetric birth and death (sorption-desorption) process and a rare event dominated problem due to the very nature of the sorption mechanism, the more active sites being the first to be occupied. We pointed out that these characteristics should be taken into account in the theory. We showed that to account for the power law Freundlich isotherm, one has to assume a Lévy heavy tail behavior for the temperature dependent Langmuir parameter.

As a consequence of this study we proposed an isotherm using a Weibull distribution known since as Brouers-Sotolongo (BS) isotherm which has been used among others in sorption on porous/nonporous surface interface [3], magnetic nano-particles [4], adsorption on doped nanostructures [5], on activated carbon produced from natural products [6]-[8], algae [9]-[11], soils and natural wastes [12] for water treatment [9] [13], biosorption and biodegradation, food contamination [14] as well as medical applications such as the chemical immobilization of bacteriophages on surfaces [15] [16].

The present paper is a extension of some of the ideas developed in our previous works. We will take advantage of the recent progress in the statistical theory of complex and deterministic chaotic systems. We will show that many of the isotherms used in the literature, especially in the treatment of water, form a subfamily of the $B u r r_{X I}$ distribution. This will lead us to propose a generalization (GBS) of the BS isotherm replacing the exponential in the Weibull function by a deformed exponential used now in the formulation of the nonextensive thermodynamics [17] and other complex systems theories. The same technique has helped us to elucidate the universality of relaxation in disordered systems [18] [19] and formulate a fractional-time kinetics for $n$-order reaction systems [20]. As we will show, many of the isotherms used in the literature can be obtained by giving well defined values to the parameters of this generalized isotherm.

\section{The Burr XII Distribution Function}

If we view the isotherm as a cumulative distribution function we can write the isotherms in the following forms:

$$
\tilde{\Theta}(P(C))=\frac{\Theta(P(C))}{\Theta_{\max }}=\int_{0}^{P(C)} \theta(p(c)) \mathrm{d} p(\mathrm{~d} c) .
$$

In Equation (1), $\theta(p(c))$ is the relative sorbed quantity as the pressure or concentration are increased in the gas or liquid phase in appropriate units. The quantity $\Theta_{\max }$ is the maximum sorption capacity in appropriate units. The $p(c)$ are supposed to be related thermodynamically to a sorption energy variable $e$ :

$$
p(c) \propto \exp (-e / R T) .
$$

In an heterogeneous system, as we increase the pressure or the concentration, the most active sites with the highest sorption energy are first occupied until complete saturation. With a change of variable, one can write 


$$
\tilde{\Theta}(P)=\int_{\Delta(P)} \theta(e) \mathrm{d} e
$$

where $\Delta(P)$ is the range of energies involved at pressure $P$ and $\theta(e)$ is an energy dependent properly normalized distribution function. This second formulation (Equation (3)) has been used to determine an empirical formula for the sorption energy distribution [21]-[24]. In the following the variables $p$ or $c$ will be denoted by the greek letter $\varkappa$.

We will now demonstrate that if we choose for $\tilde{\Theta}(\varkappa)$, the $B u r r_{X I I}$ cumulative distribution function (cdf) many of the physically sound isotherms used in the literature to generalize the Freundlich formula can be recovered and a new generalized isotherm can be proposed as a synthesis of the efforts of a few generations.

In probability theory and statistical sciences, the Burr $_{X I I}$ distribution is a continuous probability distribution for a non-negative random variable [25]. It is also known in econometrics as the Singh-Maddala distribution [26] where it has been used as a generalization of the Pareto distribution for the graduation over the whole range of incomes and is used to measure the level of inequality.

The Burr $_{X I I}$ distribution is a member of a system of continuous cumulative distribution (cdf) functions introduced by I. Burr in 1942 [25]. It has the form:

$$
F_{B}(x, a, b, c)=1-\left[1+c\left(\frac{x}{b}\right)^{a}\right]^{-1 / c}
$$

where $a, b, c$ are positive parameters. Its normalized probability density function $(p d f) f_{B}(x, a, b, c)$ is obtained from

$$
\begin{aligned}
\mathrm{d} F_{B}(x, a, b, c) & =f_{B}(x, a, b, c) \mathrm{d} x=\frac{a}{b}\left(\frac{x}{b}\right)^{\alpha-1}\left[1+c\left(\frac{x}{b}\right)^{a}\right]^{-1-1 / c} \mathrm{~d} x \\
\mathrm{~d} F_{B}\left(x^{a}, a, b, c\right) & =f_{B}\left(x^{a}, a, b, c\right) \mathrm{d} x^{a}=\left(\frac{1}{b}\right)^{a}\left[1+c\left(\frac{x}{b}\right)^{a}\right]^{-1-1 / c} \mathrm{~d} x^{a} .
\end{aligned}
$$

In previous papers [18] [19], we have shown how it could be derived from the maximum entropy principle using a generalization of the non-extensive Tsallis entropy with appropriate constraints. However more recently, it has been shown that it can be derived more naturally from the classical Boltzmann-Gibbs entropy with appropriate generalization of the moments constraints (see Section 9).

The cumulative distribution functions belonging to the Burr family are solution of the general differential equation

$$
\frac{\mathrm{d} F(x)}{\mathrm{d}(x)}=f(x)=g(x) F(x)(1-F(x))
$$

where $F(x)$ and $g(x)$ are continuous functions defined in specific domains. This differential equation describes a birth and death function modulated by a $g(x)$ function which applied to a particular problem depends on the nature of the phenomena and the influence of the environment. The first and most studied of these differential equations is the famous Verhulst logistic equation introduced in 1845 [27] to mimic and calculate population dynamics. In that case, $g(x)=1$ and its solution is

$$
F_{V}(x)=1 /(1+\exp (-x)) .
$$

In its discrete form it has been one of the first model of deterministic chaos [28].

For the Burr $_{X I I} c d f$, the twelfth one in the family, the function $g(x)=\tilde{g}_{B}(x) / x$ has the form of an hyperbolic type function, the function, $\tilde{g}_{B}(x)$ varying smoothly between $a$ and $a / c$.

$$
\begin{aligned}
& g(x)=\tilde{g}_{B}(x) / x \text { where } \tilde{g}_{B}(x)=\frac{\left(a(x / b)^{a}\right)}{\left(1+c(x / b)^{a}\right)\left(1-\left(1+c(x / b)^{a}\right)^{(-1 / c)}\right)} \\
& \tilde{g}_{B}(x) \rightarrow a \text { when } x \rightarrow 0 \text { and } \tilde{g}_{B}(x) \rightarrow a / c \text { when } x \rightarrow \infty
\end{aligned}
$$


The Burr $_{X I I}$ distribution function has become a reference distribution in complex and non equilibrium systems as the exponential and Gaussian distributions are the reference distributions in equilibrium and non interacting systems. The "dialectic" form of its differential equation shows that it could be useful to deal with phenomena like for instance epidemic propagation, population evolution, kinetics of complex reactions, economic evolution, pharmacokinetic, cancer remission and obviously sorption-desorption. It has been used extensively these last years in a variety of chaos, nonlinear and nonequilibrium problems in quasi all fields of pure and applied sciences including natural phenomena, meteorology, hydrology, earthquake, economy, sociology and medicine.

An other interesting feature of the Burr $_{X I I}$ distribution is the existence of two power laws tails, one for $x \rightarrow 0$ with exponent $a$ and one for $x \rightarrow \infty$ with exponent $\mu=a / c$. It has a limited number of finite moments depending on the value of $\mu$. When $0<\mu<1$, it has a heavy tail and belongs to the basin of attraction of the family of stable Lévy distributions. It is to say, it has some peculiar properties which have interesting consequences. Lévy functions do not obey the traditional central limit theorem and an expectation value of $x$ cannot be defined. For higher values of $\mu$ the average value increases with the number of observations following a well defined power law [29].

\section{The Subfamily of the BurrxII Distribution and the Associated Isotherms}

The Burr $_{X I I}$ function (Equation (4)) can generate a sub-family of $c d f$ distributions if one gives particular values to the two parameters $a$ and $c$ in $F_{B}(x, a, b, c)$. The case $a=1$ and $c=0$ is simply the exponential function. Some of these functions coincide with the form of well known empirical isotherms:

- For $c=0$

$$
\begin{gathered}
F_{B}(x, a, b, 0)=F_{W}(x, a, b)=1-\operatorname{Exp}\left(-\left(\frac{x}{b}\right)^{a}\right) \\
\tilde{g}_{W}(x \rightarrow 0) \rightarrow a \text { and } \tilde{g}_{W}(x \rightarrow \infty) \rightarrow \infty .
\end{gathered}
$$

This is a Weibull distribution. The corresponding isotherm in the sorption literature is known as the BrouersSotolongo (BS) isotherm:

$$
\tilde{\Theta}_{B S}(\varkappa)=\left[1-\operatorname{Exp}\left(-\left(\frac{\varkappa}{b}\right)^{a}\right)\right] .
$$

If $\varkappa \ll b$, one gets the Freundlich isotherm

$$
\Theta_{F}(\varkappa)=K_{F} \varkappa^{a} .
$$

If moreover one puts $a=1$ in Equation (12), one gets the Jovanovic isotherm [30]

$$
\tilde{\Theta}_{J}(\varkappa)=\left[1-\operatorname{Exp}\left(-\left(\frac{\varkappa}{b}\right)\right)\right] \text {. }
$$

- For $c=1$, one has:

$$
\begin{gathered}
F_{B}(x, a, b, 1)=F_{H S}(x, a, b)=\frac{\left(\frac{x}{b}\right)^{a}}{1+\left(\frac{x}{b}\right)^{a}}=1-\left[1+\left(\frac{x}{b}\right)^{a}\right]^{-1} \\
f_{B}(x, a, b, 1)=f_{H S}(x, a, b)=\frac{a}{x} \frac{\left(\frac{x}{b}\right)^{a}}{\left(1+\left(\frac{x}{b}\right)^{a}\right)^{2}} ; \quad g_{H S}(x, a, b)=a x^{-1}
\end{gathered}
$$

which is called in probability theory the loglogistic function. The corresponding isotherms are the Hill, the 
Langmuir-Freundlich and Sips isotherms

$$
\tilde{\Theta}_{L F}(\varkappa)=\frac{\left(\frac{\varkappa}{b}\right)^{a}}{1+\left(\frac{\varkappa}{b}\right)^{a}}=1-\left[1+\left(\frac{\varkappa}{b}\right)^{a}\right]^{-1} .
$$

- If both $a$ and $c$ are equal to 1 :

$$
F_{B}(x, 1, b, 1)=F_{H S}(x, 1, b)=F_{L}(x, b)=\frac{x}{b+x}=1-\left[1+\left(\frac{x}{b}\right)\right]^{-1}
$$

the corresponding isotherm is the Langmuir isotherm.

$$
\tilde{\Theta}_{L}(\varkappa)=\frac{\varkappa}{b+\varkappa} .
$$

As discussed in [1], the exponent $a$ is related to the width and shape of the sorption energy distribution which itself depends on the heterogeneity of the substrate. In Section 8 we will show that it defines an effective temperature $T^{*}=T / a$.

In the isotherms we have just reviewed, the exponent $a$ is supposed to be constant and do not change with the evolution of the sorbed quantity $\Theta$. This is a restrictive assumption. An isotherm derived from the full Burr $_{X I I}$ would allow the characteristic exponent to vary slowly from $a$ to $a / c$. Therefore quite naturally a more realistic isotherm based on the full Burr $r_{X I}$ distribution can be proposed:

$$
\tilde{\Theta}_{G B S}(\varkappa)=1-\left[1+c\left(\frac{\varkappa}{b}\right)^{a}\right]^{-1 / c} \text {. }
$$

This generalized BS isotherm has a unified character since it contains the Langmuir, the Freundlich-Langmuir, the Hill and the Sips isotherm and as we will see in the next section, the Generalized Freundlich-Langmuir and the Toth isotherms. The GBS isotherm can be written in a more compact form

$$
\tilde{\theta}_{G B S}(\varkappa)=\left\{1-\left[1+c\left(\frac{\varkappa}{b}\right)^{a}\right]^{-1 / c}\right\}=1-\exp _{c}\left(-\left(\frac{\varkappa}{b}\right)^{a}\right) .
$$

We have used the definition of the deformed exponential function introduced in mathematics in the XIX century and appearing to day in the theory of many complex systems

$$
\begin{gathered}
\exp _{c}(x)=(1-c x)^{-\frac{1}{c}} \text { if } 1-c x>0,0 \text { otherwise } \\
\log _{c}(x)=\frac{1-x^{-c}}{c} \text { with } \exp _{c}\left(\log _{c}(x)\right)=\log _{c}\left(\exp _{c}(x)\right)=x .
\end{gathered}
$$

When $c=0$, one recovers the usual exponential. In the nonequilibriun thermodynamic literature $c=q-1$ where $q$ is the nonextensive (nonadditive) entropy index [17]. In the complex reaction literature, $c=n-1$, where $n$ is the effective fractional reaction order. In the extreme value theory $c=\xi$, the shape parameter of the distribution. We recover the $B S$ isotherm $\theta_{B S}$ if $c=0$ and the Hiil-Sips adsorption for $c=1$.

This new isotherm has four parameters $\theta_{\max }, a, b$, and $c$ which have simple physical interpretation: $\theta_{\max }$ is the maximum saturation sorbed quantity, $a$ is the Freundlich exponent which is related to the width and shape of the sorption energy and is a measure of the distribution. When $a<1$, it can be related to the selfsimilar (fractal) properties at the micro- and meso-scopic scale. For $a>1$, it has been interpreted as the manifestation of a multi-molecular site sorption [31]. The coefficient $0 \leq c \leq 1$ is related to the cluster organization of the system. A large $c$ corresponds to a strong clustering organization [32]. The coefficient $b$ is a $T$ dependent scale parameter and combined with $a$ and $c$ allows the calculation of all the quantities characterizing the statistical distribution: expectation, variance and moments, median, quantiles and some other coefficients which measure quantitatively the way the sorption depends on the concentration or the pressure. These useful 
expressions for the analysis of isotherms are derived in the appendix. The value $a=1$ separates the distributions defining the isotherms in two groups. For $a \leq 1$ and this includes the Langmuir isotherm, the $p d f$ is $L$-shaped while for $a>1$, it is unimodular. This has a strong influence on the nature of the sorption. We will show also in the appendix that the quantity $b^{a}$ is directly related to specific moment of the probability distribution. Finally when $(a / c)=\mu$ is $<1$ it is the heavy tail (Lévy) exponent which controls the saturation behavior of the sorption curve.

\section{The Generalized Freundlich-Langmuir and Toth Isotherms}

A two exponents isotherm (GFL) generalizing the Freundlich-Langmuir (Hill, Sips) isotherm was proposed by Marczewski and Jaroniec [33].

$$
\tilde{\theta}_{G F L}(\varkappa)=\left(\frac{\left(\frac{\varkappa}{b}\right)^{n}}{1+\left(\frac{\varkappa}{b}\right)^{n}}\right)^{\frac{m}{n}} .
$$

The corresponding $c d f$ function

$$
F_{G}(x)=\left(\frac{\left(\frac{x}{b}\right)^{n}}{1+\left(\frac{x}{b}\right)^{n}}\right)^{\frac{m}{n}}=\left(1+\left(\frac{x}{b}\right)^{-n}\right)^{-\frac{m}{n}}
$$

has the characteristics of a $c d f \quad F_{G}(0)=0, \quad F_{G}(\infty)=1$

It appears that $F_{G}(x)$ has the form of a Dagun function [34] used concurrently with the Burr $_{X I}$ equation in econometrics. It can be related to the Burr ${ }_{X I I}$ function by a simple change of variables. This will allow us to relate the isotherms obtained from the GFL isotherm form (20) to the ones already derived.

As $F_{B}(x), F_{G}(x)$ is also the solution of a first order differential equation. Indeed one has

$$
\frac{\mathrm{d} F_{G}(x)}{\mathrm{d} x}=\frac{m b\left(\frac{\left(\frac{x}{b}\right)^{n}}{1+\left(\frac{x}{b}\right)^{n}}\right)^{\frac{m}{n}}}{x\left(1+\left(\frac{x}{b}\right)^{n}\right)}=\frac{m b}{x}\left(\frac{x}{b}\right)^{-n} F_{G}(x)^{\frac{n}{m}+1} .
$$

We have moreover:

$$
\text { for } x \rightarrow 0, \frac{\mathrm{d} F_{G}(x)}{\mathrm{d} x} \rightarrow m b\left(\frac{x}{b}\right)^{m-1} \text { and for } x \rightarrow \infty, \frac{\mathrm{d} F_{G}(x)}{\mathrm{d} x} \rightarrow m b\left(\frac{x}{b}\right)^{-n-1} .
$$

These asymptotic behaviors which are supposed to be the same as the ones of $\frac{\mathrm{d} F_{B}(x)}{\mathrm{d} x}$ gives the relations between the exponents of the two formulations.

$$
m=a, m / n=c, n=a / c=\mu
$$

Starting from the $G L F$ isotherm equation, one can recover some of the empirical isotherms: for $m=n=1$, the Langmuir isotherm, for $m=n$, the Langmuir-Freundlich or Hill isotherm. For $n=1$, the Sips isotherm and for $m=1$ the Toth [35] isotherm. The first ones belong to the subfamily of the Burr ${ }_{X I I}$ subfamily isotherms and have been already considered. The Toth isotherm is applicable only for $m=a=1$ and for $c=1 / n$. We will see now how the Marczewski and Jaroniec GLF is linked to the Burr $r_{X I I}$ function using the relations between the two probability functions. 


\section{Dagum Distribution versus Burr ${ }_{X I I}$ Distribution}

The Burr ${ }_{X I I} c d f$ and $p d f$ functions (Equations (4), (5)) can be written

$$
\begin{gathered}
F_{B}(x, a, \tilde{b}, c)=1-\left[1+\left(\frac{x}{\tilde{b}}\right)^{a}\right]^{-1 / c} \text { with } \tilde{b}=b\left(c^{-\frac{1}{a}}\right) \\
f_{B}(x, a, \tilde{b}, c)=\frac{a}{\tilde{b} c}\left(\frac{x}{\tilde{b}}\right)^{\alpha-1}\left[1+\left(\frac{x}{\tilde{b}}\right)^{a}\right]^{-1-1 / c} .
\end{gathered}
$$

If we make the change of variables $x \rightarrow 1 / x$ and $\tilde{b} \rightarrow 1 / \tilde{b}$ in (Equations (29) and (30)), we get the Dagum $c d f$ and $p d f$ :

$$
\begin{gathered}
F_{D}(x, a, \tilde{b}, c)=\left[1+\left(\frac{x}{\tilde{b}}\right)^{-a}\right]^{-1 / c} \\
f_{D}(x, a, \tilde{b}, c)=-\frac{a}{\tilde{b} c}\left(\frac{x}{\tilde{b}}\right)^{-\alpha-1}\left[1+\left(\frac{x}{\tilde{b}}\right)^{-a}\right]^{-1-1 / c} .
\end{gathered}
$$

Therefore one has the relation

$$
\begin{aligned}
& F_{B}(x, a, \tilde{b}, c)=1-F_{D}(1 / x, a, 1 / \tilde{b}, c) ; \quad f_{B}(x, a, \tilde{b}, c)=-f_{D}(1 / x, a, 1 / \tilde{b}, c)\left(1 / x^{2}\right) \\
& \text { and } F_{D}(x, a, \tilde{b}, c)=1-F_{B}(1 / x, a, 1 / \tilde{b}, c) ; \quad f_{D}(x, a, \tilde{b}, c)=-f_{B}(1 / x, a, 1 / \tilde{b}, c) x^{2} .
\end{aligned}
$$

The relation between the Generalized Freundlich-Langmuir function and the Burr ${ }_{X I I}$ function can be written using the previous results:

$$
\left(\frac{\left(\frac{x}{\tilde{b}}\right)^{n}}{1+\left(\frac{x}{\tilde{b}}\right)^{n}}\right)^{\frac{m}{n}}=\left(1+\left(\frac{\tilde{b}}{x}\right)^{n}\right)^{-\frac{m}{n}}=F_{D}\left(x, n, \tilde{b}, \frac{m}{n}\right)=1-F_{B}\left(\frac{1}{x}, n, \frac{1}{\tilde{b}}, \frac{n}{m}\right), \tilde{b}=b\left(\frac{m}{n}\right)^{-\frac{1}{n}} .
$$

This allows the GLF isotherm and the Toth [35] isotherm as well as the equivalent Oswin isotherm [36] used in food industry to be part of the Burr $r_{X I}$ isotherm family.

The others empirical isotherms [37] [38] correspond to couples of values $m$ and $n$ in the general form (Equation (25)) which give non physical asymptotic behavior and therefore cannot be used over the whole range of concentration or pressure They might give excellent fit over a limited range of data, like the popular RedlichPeterson isotherm [37], but cannot give reliable information over the whole sorption process. The same is true for the Freundlich isotherm. In our opinion, as a logical consequence of our work these isotherms should be discarded since we dispose now, with the unified GBS form (Equations (20), (21)), of a four parameter isotherm with a solid theoretical and physical foundation.

We can now derive quite simply the shape of the sorption energy distribution giving rise to the various isotherms we have just derived.

\section{Sorption Energy Distributions}

As we already discussed in a previous publication, starting from the thermodynamic relation

$$
\varkappa=\exp (-e / R T)
$$

and using the probability theory relation

$$
\left|f_{p}(\varkappa) \mathrm{d} \varkappa\right|=\left|f_{E}(e) \mathrm{d} E\right|
$$

it is possible to calculate the sorption energy distribution corresponding to each isotherm. As discussed later, this sorption energy $e$ is the energy which governs the macroscopic thermodynamic properties of the system. It is 
not the microscopic site energies resulting from the atomic and molecular interactions.

In that way we have obtained the following results:

- For the proposed GBS. isotherm derived from the Burr $X_{X I}$ distribution function:

$$
\phi_{G B S}(e)=\frac{a}{R T}\left(b^{-a} \exp (-a e / R T)\right)\left(1+c\left(b^{-a} \exp (-a e / R T)\right)\right)^{-1-1 / c} .
$$

The other distributions can be obtained easily:

- For $c \rightarrow 0$, we have the distribution corresponding to the BS isotherm

$$
\phi_{B S}(e)=\frac{a}{R T}\left(b^{-a} \exp (-a e / R T)\right) \exp \left(b^{-a} \exp (-a e / R T)\right) .
$$

- For $c \rightarrow 1$, we have the distribution corresponding to the Hill-Sips isotherm:

$$
\phi_{H}(e)=\frac{a}{R T}\left(b^{-a} \exp (-a e / R T)\right)\left(1+\left(b^{-a} \exp (-a e / R T)\right)\right)^{-2} .
$$

It is worth noticing that the BS. distribution has the form of the Gumbel [39] [40] (maximum) extreme value probability distribution function

$$
\begin{gathered}
\phi_{G}(e)=\frac{1}{\beta} \operatorname{Exp}\left(-\left(\frac{e-\mu}{\beta}\right)\right) \operatorname{Exp}\left(-\operatorname{Exp}\left(\frac{e-\mu}{\beta}\right)\right) \\
\Phi_{G}(E)=\operatorname{Exp}\left(-\operatorname{Exp}\left(-\left(\frac{E-\mu}{\beta}\right)\right)\right) \\
\text { with } \beta=R T / a \text { and } \mu=-R T \log b .
\end{gathered}
$$

The standard deviation of this function is well known

$$
\beta \pi / \sqrt{6}=(k T / a) \pi / \sqrt{6}
$$

confirming the conclusions of reference [1] about the physical signification of the exponent $a$.

The function $\phi_{G B S}(e)$ corresponding to the new proposed GBS isotherm is one member of the family of generalized Gumbel functions.

$$
\begin{gathered}
\phi_{G B S}(e)=\frac{a}{R T}\left(b^{-a} \exp (-a e / R T)\right)\left(1+c\left(b^{-a} \exp (-a e / R T)\right)\right)^{-1-1 / c} \\
\Phi_{G B S}(E)=\operatorname{Exp}_{c}\left(-\operatorname{Exp}\left(-\left(\frac{E-\mu}{\beta}\right)\right)\right) \\
\text { with } \beta=R T / a \quad \text { and } \mu=-R T \log b
\end{gathered}
$$

It is the symmetric of the Fisher-Tippett [40] [41] generalized extreme value cumulative distribution

$$
F_{F T}(E)=\operatorname{Exp}\left(-\operatorname{Exp}_{c}\left(-\left(\frac{E-\mu}{\beta}\right)\right)\right) .
$$

It is worth noticing that this last GEV function (Equation (45)) could have been obtained by using the $B S$ isotherm (Equation (12)) and a $c$-deformed thermodynamic exponential (see Equation (22)) expression

$$
\varkappa_{i}=\operatorname{Exp}_{c}\left(-e_{i} / R T\right)=\left(1-c\left(e_{i} / R T\right)\right)^{-1 / c} .
$$

To be complete we have calculated the energy distributions corresponding to the Freundlich-Langmuir isotherm

$$
\phi_{F L}(e)=\frac{m}{R T} \frac{b^{-m} \exp (-m e / R T)}{\left(1+b^{-n} \exp (-n e / R T)\right)^{\frac{m}{n}+1}} .
$$


If $m=n$ (Hill, Sips)

$$
\phi_{H}(e)=\frac{n}{R T} \frac{b^{-n} \exp (-n e / R T)}{\left(1+b^{-n} \exp (-n E / R T)\right)^{2}} .
$$

If $m=1 \quad$ (Toth, Oswin)

$$
\phi_{T}(e)=\frac{1}{R T} \frac{b^{-1} \exp (-e / R T)}{\left(1+b^{-n} \exp (-n e / R T)\right)^{\frac{1}{n}+1}} .
$$

If $n=1 \quad$ (Generalized-Freundlich)

$$
f_{G F}(e)=\frac{m}{R T} \frac{b^{-m} \exp (-m e / R T)}{\left(1+b^{-1} \exp (-e / R T)\right)^{m+1}} .
$$

Some of the these distributions have been obtained earlier by various authors without reference to the probability theory and using the Cerofolini condensation approximation method [21]. Equation (41) was derived in [22], Equations (39), (50) was derived in [23] and Equation (52) in [24]. They have been used to determine numerically sorption energy distributions from isotherm data and investigate the thermodynamic nature of the sorption from the measured isotherms. The detailed calculations require assumptions on the range of sorption energy, the integrals being performed from $E_{\min }$ to $E_{\max }$ with respect to a reference energy $E_{0}$. As $a$ and $c$ tend to 1 , one recovers the Langmuir isotherm, the model with a unique sorption energy. Indeed the energy probability density (Equation (39) with $a=1$ ) is the derivative of a Fermi function and tends to a Heaviside function as $T$ tends to 0 . The corresponding pressure or concentration density function has a horizontal asymptote at the origin. Physically this means that on a homogeneous surface the pressure range over which sorption takes place (from a few percents to complete coverage) at finite temperature, will be only of one or two order of magnitude, and be narrower as $T$ decreases (and $b$ decreases), an observation already discussed by Roginskii [42].

\section{Langmuir, Hill-Sips and Brouers-Sotolongo Isotherms Obey the Maximum Entropy Principle}

Before concluding this study it is worthwhile to point out that the distribution functions giving the Langmuir, the Hill-Sips-“Langmuir-Freundlich”, the Brouers-Sotolongo and Generalized Brouers-Sotolongo can be derived maximizing the Boltzmann-Shannon entropy measure:

$$
S(x)=-\int f(x) \log f(x) \mathrm{d} x
$$

using the Lagrange multipliers methods [43] [44] with constraints generalizing the ones used for the Weibull distribution [45] by introducing a $c$-deformation of the power function

$$
x_{c}^{a}=\frac{1}{c} \ln \left(1+c(x)^{a}\right) \quad x_{c \rightarrow 0}^{a}=x^{a}
$$

in the same spirit as the deformation of the exponential function (Equation (22)). One uses the following constraints:

$$
\begin{gathered}
\int_{0}^{\infty} \ln \left(\frac{x}{b}\right)^{a} f_{G B S}(x ; a, b, c) \mathrm{d} x=-\gamma-\ln (c)-\psi(1 / c) \\
\int_{0}^{\infty}\left(\left(\frac{x}{b}\right)^{a}\right)_{c} f_{G B S}(x ; a, b, c) \mathrm{d} x=\int_{0}^{\infty} \frac{1}{c} \ln \left(1+c\left(\frac{x}{b}\right)^{a}\right) f_{G B S}(x ; a, b, c) \mathrm{d} x=1
\end{gathered}
$$

where $\gamma$ is the Euler constant and $\psi(x)$ is the BiGamma function.

For $c->0$ (Brouers-Sotolongo) and $c->1$ (Hill-Sips) and Langmuir $(c=a=1)$ these constraints can be simplified to 


$$
\begin{aligned}
& \int_{0}^{\infty} \ln \left(\frac{x}{b}\right) f_{B S}(x ; a, b, c) \mathrm{d} x=-\gamma / a \\
& \int_{0}^{\infty}\left(\frac{x}{b}\right)^{a} f_{B S}(x ; a, b) \mathrm{d} x=1
\end{aligned}
$$

which are the well known Weibull constraints and

$$
\begin{gathered}
\int_{0}^{\infty} \ln \left(\frac{x}{b}\right) f_{L}(x ; b) \mathrm{d} x=0 \\
\int_{0}^{\infty}\left(\left(\frac{x}{b}\right)^{a}\right)_{c=1} f_{L}(x ; b) \mathrm{d} x=\int_{0}^{\infty} \ln \left(1+\left(\frac{x}{b}\right)^{a}\right) f_{L}(x ; b) \mathrm{d} x=1
\end{gathered}
$$

which are the loglogistic constraints. The fact that these isotherms correspond to the maximum entropy show that they are the best and less biased isotherms when the parameters $a, b$ and $c$ can be determined experimentally and therefore should be favoured amongst all the proposed empirical formulas.

\section{Conclusions}

In this paper we have shown that a generalized isotherm having the analytical form of a Burr $_{X I I} \quad c d f$ is able to generate a whole family of empirical isotherms used in the literature to represent the sorption data of a great number of solid-gas and solid-liquid sorbate-sorbent couples. Due to the fact that the Burr $r_{X I I}$ and associated functions are used extensively in econometrics, there exists on the market efficient nonlinear fitting computing programs and the use of the GBS isotherm should make obsolete the comparison, often with questionable linear fitting, of experimental isotherms with the various approximations of this more general unified isotherm. Practically since the GBS isotherm interpolates nicely between the $B S(c=0)$ and the Hill-Sips $(c=1)$ isotherm and since the two $(a, b)$ parameters isotherms give generally a reasonably good fit, one can first try both of them and then using these partial results improve the fit with the three $(a, b, c)$ parameters $G B S$ when this is possible given the generally scarcity of data.

The statistical expressions given in the appendix allow a mathematically well defined characterization of the data. Extensions of the Burr ${ }_{X I}$ have been proposed with extra parameters. They belong to the Generalized Beta 2 distribution family and are legitimate cumulative probability functions [46]. Such an extension which might be of interest for huge number of data are irrelevant in sorption problems due to the relatively small number of experimental data.

Another important conclusion of this study is that the energy distributions giving rise to the $B S$ and $G B S$ isotherms belong to the family of extreme value distributions. This is in agreement with the stochastic theory of K. Weron et al. [18] [32] [47] which was developed for relaxation in disordered medias. What matters in highly heterogeneous media is not the detailed microscopic interactions but the extreme value distribution of interaction energies of dynamically highly correlated mesoscopic clusters (on surfaces, patches, islands). The relation between the phenomenological laws and their microscopic causes has to go through the spatio-temporal scaling properties of these intermediate cooperative regions. This representation allows to average together a large number of extreme probabilistic events to form a predictable picture of the behavior of the entire system. As a consequence, the observed tail exponents $a$ and $a / c$ and the analytic form of the equations describing the macroscopic properties are related to the extreme value cluster energy distributions. The parameter $a$ defined an effective temperature $T^{*}=T / a$ and $c=q-1$ is related to the Reyni-Tsallis entropy factor $q$. In catalysis, the appearance of an effective temperature $T^{*}$ has been traced to the conditions at which the substrate was prepared and annealed. The active centers regarded as defects once in thermal equilibrium at temperature $T^{*}$ are "frozen" by sudden cooling (quenching) [42]. More generally an effective temperature $T^{*} \neq T$ expresses the fact that, due to the frustrations induced by the geometry and the interactions, the couple sorbate-sorbent is not in thermal equilibrium at the experimental temperature $T$.

Two last remarks have to be made on the range of applicability of the results of this paper. One has to emphasize that it deals with one aspect of sorption i.e. the generalization of the Langmuir isotherm to highly heterogeneous surfaces and solid-liquid interfaces and in some cases of complex composition of sorbates and 
sorbent. It concerns in particular most works done in water and air decontamination research with pure or treated natural products.

The sorption of simple molecules on smooth surfaces and well defined rough surfaces [48] [49] does not necessarily necessitate an elaborate treatment as used in this paper and the analysis of its isotherms can bring some partial information on the microscopic properties of the surface. In many more complex systems, other phenomena such as wetting, capillarity condensation in pores [50], as well as diffusion, volume condensation and multi-reactions effects might have to be considered. In those cases, more specific isotherm formulas have to be used [51]. One should also be conscious that the analysis of data with the GBS, Hill-Sips and BS isotherms is relevant only when applied to complete sets of data until saturation.

\section{References}

[1] Brouers, F., Sotolongo-Costa, O., Marquez, F. and Pirard, J.P. (2005) Microporous and Heterogeneous Surface Adsorption Isotherms Arising from Lévy Distributions. Physica A, 349, 271-282.

http://dx.doi.org/10.1016/j.physa.2004.10.032

[2] Tien, C. (2008) Remarks on Adsorption Manuscripts Revised and Declined: An Editorial. Journal of Hazardous Materials, 150, 2-3. http://dx.doi.org/10.1016/j.jhazmat.2007.04.015

[3] Ncibi, M.C., Altenor, S., Steffen, M., Brouers, F. and Gaspard, S. (2008) Modelling Simple Compound Adsorption onto Porous and Non-Porous Sorbents Using a Deformed Weibull Exponential Isotherm. Chemical Engineering, 145, 196-202. http://dx.doi.org/10.1016/j.cej.2008.04.001

[4] Madrakian, T., Afkhami, F. and Ahmadi, M. (2012) Adsorption and Kinetic Studies of Different Organic Dyes onto Magnetic Nanoparticles Loaded Tea Waste and Removal of Them from Wastewater Samples. Spectrochimica Acta A, 99, 102-109. http://dx.doi.org/10.1016/j.saa.2012.09.025

[5] Azizian, S. and Baghery, M. (2014) Enhanced Adsorption of $\mathrm{Cu}^{2+}$ from Aqueous Solution by Ag Doped Nano-Structured ZnO. Journal of Molecular Liquids, 196, 198-203. http://dx.doi.org/10.1016/j.molliq.2014.03.043

[6] Vargas, A.M.M., Cazela, A.J., Kunita, M.H. and Silva, T.L. (2011) Adsorption of Methylene Blue on Activated Carbon from Flamboyant Pads: Study of Adsorption Isotherms and Kinetic Models. Chemical Engineering Journal, 168, 722-730. http://dx.doi.org/10.1016/j.cej.2011.01.067

[7] Altenor, S., Carene, B., Enmanuel, E., Lamber, J., Ehrharat, J.J. and Gaspard, S. (2009) Adsorption Studies of Methylene Blue and Phenol onto Vetiver Roots Activated Carbon Prepared by Chemical Activation. Journal of Hazardous Materials, 165, 1029-1039. http://dx.doi.org/10.1016/j.jhazmat.2008.10.133

[8] Hejazifar, M. and Azizian, S. (2012) Adsorption of Cationic and Anionic Dyes onto the Activated Carbon Prepared from Grapevine Rhytidome. Journal of Dispersion Science and Technology, 33, 846-853. http://dx.doi.org/10.1080/01932691.2011.579861

[9] Ncibi, M.C., Majoub, B., Steffen, M., Brouers, F. and Gaspard, S. (2009) Sorption Dynamic Investigation of Chromium (VI) onto Posidonia Oceanica Fibres: Kinetic Modelling Using New Generalized Fractal Equation. Biochemical Engineering Journal, 46, 141-146. http://dx.doi.org/10.1016/j.bej.2009.04.022

[10] Altenor, S., Ncibi, M.C., Emmanuel, E. and Gaspard, S. (2012) Textural Characteristic Physiochemical Properties and Adsorption Efficiencies of Caribbean Alga. Turbinaria turbinata and Its Derived Carbonaceous Materials for Water Treatment Application. Biochemical Engineering Journal, 67, 35-44. http://dx.doi.org/10.1016/j.bej.2012.05.008

[11] Hamissa, A.M.B., Brouers, F., Ncibi, M.C. and Steffen, M. (2013) Kinetic Modeling and Methylène Sorption onto Agava Americana Fibres: Fractal Kinetic and Regeneration Studies. Separation Science and Technology, 48, 28342842. http://dx.doi.org/10.1080/01496395.2013.809104

[12] Salleh, M., Mahmou, D., Karim, W. and Idris, A. (2011) Cationic and Anionic Dye Adsorption by Agricultural Solid Wastes: A Comprehensive Review. Desalination, 280, 1-13. http://dx.doi.org/10.1016/j.desal.2011.07.019

[13] Figaro, S., Avril, J.P., Brouers, F., Ouensanga, A. and Gaspad, S. (2009) Adsorption Studies of Molasse's Wastewaters on Activated Carbon: Modelling with a New Fractal Kinetic Equation and Evaluation of Kinetic Models. Journal of Hazardous Materials, 161, 649-656. http://dx.doi.org/10.1016/j.jhazmat.2008.04.006

[14] Singh, A., Arutyunov, D., Szymanski, C.M. and Evoy, S. (2012) Bacteriophage Based Probes for Pathogen Detection. Analyst, 137, 3405-3421. http://dx.doi.org/10.1039/c2an35371g

[15] Naidoo, R., Singh, A., Arya, S.K., Beadle, B., Glass, N., Tanha, J., Szymanski, C.M. and Evoy, S. (2012) SurfaceImmobilization of Chromatographically Purified Bacteriophages for the Optimized Capture of Bacteria. Bacteriophage, 2, 15-24. http://dx.doi.org/10.4161/bact.19079

[16] Singh, A., Poshtiban, S. and Evoy, S. (2013) Recent Advances in Bacteriophage Based Biosensors for Food-Borne Pathogen Detection. Sensors, 13, 1763-1786. http://dx.doi.org/10.3390/s130201763 
[17] Tsallis, C. (2009) Nonadditive Entropy and Nonextensive Statistical Mechanics. An Overview after 20 Years. Brazilian Journal of Physics, 39, 337-357. http://dx.doi.org/10.1590/S0103-97332009000400002

[18] Brouers, F., Sotolongo-Costa, O. and Weron, K. (2004) Burr, Lévy, Tsallis. Physica A, 344, 409-416. http://dx.doi.org/10.1016/j.physa.2004.06.008

[19] Brouers, F. and Sotolongo-Costa, O. (2005) Relaxation in Heterogeneous Systems: A Rare Events Dominated Phenomenon. Physica A, 356, 359-374. http://dx.doi.org/10.1016/j.physa.2005.03.029

[20] Brouers, F. and Sotolongo-Costa, O. (2006) Generalized Fractal Kinetics in Complex Systems (Application to Biophysics and Biotechnology). Physica A, 368, 165-175. http://dx.doi.org/10.1016/j.physa.2005.12.062

[21] Cerofolini, G.F. (1974) Localized Adsorption on Heterogeneous Surfaces. Thin Solid Films, 23, 129-152. http://dx.doi.org/10.1016/0040-6090(74)90235-1

[22] Jaroniec, M. and Piotowska, J. (1986) Isotherm Equations for Adsorption on Heterogeneous Microporous Solids. Monatshefte für Chemie/Chemical Monthly, 117, 7-19.

[23] Yaghi, N.Z. (2007) Iron Oxide Based Materials for the Removal of Copper from Drinking Water-A Study of Freundlich Adsorption Isotherms, Site Energy Distributions and Energy Frequency Distributions. Master's Thesis, Chalmers University of Technology, Göteborg.

[24] Kumar, K.V., Monteiro de Castro, M., Martinez-Escandell, M., Molina-Sabio, M. and Rodriguez-Reinoso, F. (2011) A Site Energy Distribution Function from Toth Isotherm for Adsorption of Gases on Heterogeneous Surfaces. Physical Chemistry Chemical Physics, 13, 5753-5759. http://dx.doi.org/10.1039/c0cp00902d

[25] Burr, I.W. (1942) Cumulative Frequency Functions. The Annals of Mathematical Statistics, 13, 215-232. http://dx.doi.org/10.1214/aoms/1177731607

[26] Maddala, G.S. (1983) Limited-Dependent and Qualitative Variables in Econometrics. Cambridge University Press, Cambridge.

[27] Verhulst, P.F. (1845) Recherches mathématiques sur la loi d’accroissement de la population. Nouveaux Mémoires de l'Académie Royale des Sciences et Belles-Lettres de Bruxelles, 18, 14-54.

[28] Ausloos, M. and Dirickx, M. (2005) The Logistic Map and the Road to Chaos. Springer, Berlin.

[29] Sornette, D. (2004) Critical Phenomena in Natural Sciences. 2nd Edition, Springer, Berlin.

[30] Jovanovic, D.S. (1969) Physical Adsorption of Gazes. Kolloid-Zeitschrift und Zeitschrift für Polymere, 235, 12141225.

[31] Monod, J., Wyman, J. and Changeux, J.P. (1965) On the Nature of Allosteric Transitions: A Plausible Model. Journal of Molecular Biology, 12, 88-118. http://dx.doi.org/10.1016/S0022-2836(65)80285-6

[32] Stanislavsky, A. and Weron, K. (2013) Is There a Motivation for a Universal Behaviour in Molecular Populations Undergoing Chemical Reactions? Physical Chemistry Chemical Physics, 15, 15595-15601. http://dx.doi.org/10.1039/c3cp52272e

[33] Marczewski, A.W. and Jaroniec, M. (1983) A New Isotherm Equation for Single-Solute Adsorption from Dilute Solutions on Energetically Heterogeneous Solids. Monatshefte für Chemie/Chemical Monthly, 114, 711-715. http://dx.doi.org/10.1007/BF01134184

[34] Dagum, C. (1977) A New Model of Personal Income-Distribution-Specification and Estimation. Economie Appliquée, 30, 413-437.

[35] Toth, J. (1995) Uniform Interpretation of Gas/Solid Adsorption. Advances in Colloid and Interface Science, 55, 1-239. http://dx.doi.org/10.1016/0001-8686(94)00226-3

[36] Oswin, C.R. (1946) The Kinetics of Package Life III. The Isotherm. Journal of the Society of Chemical Industry, 65, 419-421. http://dx.doi.org/10.1002/jctb.5000651216

[37] Redlich, O. and Peterson, D.L. (1959) A Useful Adsorption Isotherm. Journal of Physical Chemistry, 63, 1024. http://dx.doi.org/10.1021/j150576a611

[38] Müller, G., Radke, C.J. and Prausnitz, J.M. (1985) Adsorption of Weak Organic Electrolytes from Dilute Aqueous Solution onto Activated Carbon. Part I. Single-Solute Systems. Journal of Colloid and Interface Science, 103, 466-483. http://dx.doi.org/10.1016/0021-9797(85)90123-7

[39] Gumbel, E.J. and Lieblein, J. (1954) Statistical Theory of Extreme Values and Some Practical Applications: A Series of Lectures (Vol. 33). US Government Printing Office, Washington.

[40] Coles, S. (2001) An Introduction to Statistical Modeling of Extreme Values. Springer Verlag, Berlin. http://dx.doi.org/10.1007/978-1-4471-3675-0

[41] Fischer, R.A. and Tippett, L.H.C. (1928) Limiting Forms of the Frequency Distribution of the Largest or Smallest Member of a Sample. Mathematical Proceedings of the Cambridge Philosophical Society, 24, 180-190. 
http://dx.doi.org/10.1017/S0305004100015681

[42] Young, D.M. and Crowell, A.D. (1962) Physical Adsoption of Gases, Chapter 7, Buttenwerks, London.

[43] Papalexiou, S.M. and Koutsoyiannis, D. (2012) Entropy Based Derivation of Probability Distributions: A Case Study to Daily Rainfall. Advances in Water Resources, 45, 51-57. http://dx.doi.org/10.1016/j.advwatres.2011.11.007

[44] Park, S.Y. and Bera, A.K. (2009) Maximum Entropy Autoregressive Conditional Heteroskedasticity Model. Journal of Econometrics, 150, 219-230. http://dx.doi.org/10.1016/j.jeconom.2008.12.014

[45] Kapur, J.N. (1993) Maximum Entropy Models in Science and Technology. Wiley Eastern Limited.

[46] McDonald, J.B. (1984) Some Generalized Functions for the Size Distribution of Income. Journal of the Econometric Society, 52, 647-663. http://dx.doi.org/10.2307/1913469

[47] Hetman, P. and Weron, K. (2004) Extreme-Value Approach to the Tsallis' Superstatistics. Acta Physica Polonica B, 35, 1375-1386.

[48] Sahouli, B., Blacher, S. and Brouers, F. (1997) Applicability of the Fractal FHH Equation. Langmuir, 13, $4391-4394$. http://dx.doi.org/10.1021/la962119k

[49] Blacher, S., Pirard, R., Pirard, J.P., Sahouli, B. and Brouers, F. (1997) On the Texture Characterization of Mixed $\mathrm{SiO}_{2}-\mathrm{ZrO}_{2}$ Aerogels Using the Nitrogen Adsorption-Desorption Isotherms: Classical and Fractal Methods. Langmuir, 13, 1145-1149. http://dx.doi.org/10.1021/la950883l

[50] Sahouli, B., Blacher, S. and Brouers, F. (1996) Fractal Surface Analysis by Using Nitrogen Adsorption Data: The Case of the Capillary Condensation Regime. Langmuir, 12, 2872-2874. http://dx.doi.org/10.1021/la950877p

[51] Dabrowski, A. (2001) Adsorption: From Theory to Practice. Advances in Colloid and Interface Science, 93, $135-224$. http://dx.doi.org/10.1016/S0001-8686(00)00082-8

[52] Brouers, F. (2013) Sorption Isotherms and Probability Theory of Complex Systems. arXiv:1309.5340. 


\section{Appendix}

The statistical quantities of all isotherms deriving from the unified GBS isotherm will be obtained simply by giving the corresponding values to the coefficient $a, b$ and $c$ to the statistical quantities of that isotherm viewed as a $c d f$.

What we need to characterize statistically the experimental isotherms are the maximum sorbed quantity corresponding to the saturation $\Theta_{\max }$ the moments of the distribution, the pressure or concentration $\varkappa_{\max }$ corresponding to the maximum sorption rate $\left(\frac{\mathrm{d} \Theta}{\mathrm{d} \varkappa}\right)_{\max }$, the quantiles i.e. the value of $\varkappa$ corresponding to a given percentage of $\Theta_{\max }$.

We can determine the value of $\varkappa_{\max }$ when an inflexion point occurs i.e. when the second derivative of the distribution changes sign i.e. at

$$
\varkappa_{\text {max }}=b\left(\frac{a-1}{a+c}\right)^{\frac{1}{a}} \quad a>1 .
$$

For $a \leq 1, \quad \varkappa_{\max }=0$.

The expression for the $k$-th moment is

$$
\left\langle\varkappa^{k}\right\rangle=\frac{b^{k}}{c^{k / a}} \frac{\Gamma\left(1+\frac{k}{a}\right) \Gamma\left(\frac{1}{c}-\frac{k}{a}\right)}{\Gamma\left(\frac{1}{c}\right)} \quad k<\frac{a}{c}
$$

where $\Gamma$ is the Gamma function.

From Equation (62), one can calculate the expectation value $\langle\varkappa\rangle$ and the variance $\left\langle\varkappa^{2}\right\rangle-(\langle\varkappa\rangle)^{2}$.

The inverse of the Burr $_{X I I}$ function

$$
F_{B}(x)^{-1}=b\left[\frac{(1-x)^{-c}-1}{c}\right]^{\frac{1}{a}}
$$

allows us to know the pressure (or the concentration) corresponding to a given percentage of the sorbed quantity.

One can then calculate the quantile $\varkappa_{p \%}$ solution of $\tilde{\Theta}_{G B S}\left(\varkappa_{p \%}\right)=\theta_{\%}$, where $\theta_{\%}$ is the percentage of the sorbed quantity ranging from 0 to 1 . We have therefore using Equation (65):

$$
\begin{aligned}
& \varkappa_{G B S, \theta_{\%}}=b\left[\frac{\left(1-\theta_{\%}\right)^{-c}-1}{c}\right]^{1 / a} \\
& \varkappa_{H, \theta_{\%}}=b\left(\frac{\theta_{\%}}{1-\theta_{\%}}\right)^{1 / a} \\
& \varkappa_{B S, \theta_{\%}}=b\left(-\log \left(1-\theta_{\%}\right)\right)^{1 / a} .
\end{aligned}
$$

These are the values of $\varkappa$ corresponding to a given percentage $\theta_{\%}$ of the sorbed quantity.

What to do when $a \leq 1$ and an expectation value cannot be calculated? We will show that it is nevertheless possible to calculate finite characteristic quantities which can characterize the distribution.

Starting from the expression of the $k$ th moment (Equation (65)) and choosing the value

$$
k=a\left(\frac{1-c}{c}\right)
$$

the expression (64) yields 


$$
c / b^{a}=\eta=\left\langle\left[\varkappa^{a\left(\frac{1-c}{c}\right)}\right]\right\rangle^{-\frac{c}{1-c}}=\langle\rangle_{a, c} .
$$

This statistical quantity $\eta$ can be used to relate the Burr XII to a finite generalized moment which is always finite.

$$
\begin{gathered}
F_{B}(x, a, b, c)=1-\left[1+\eta x^{a}\right]^{-1 / c}=1-\left[1+\langle\rangle_{a, c} x^{a}\right]^{-1 / c} \\
f_{B}(x, a, b, c)=c^{-1}\langle\rangle_{a, c}(x)^{\alpha-1}\left[1+\langle\rangle_{a, c} x^{a}\right]^{-1-1 / c}
\end{gathered}
$$

The pdf Burr ${ }_{X I I}$ function is the normalized function which maximizes the entropy when the exponents $a$ and $c$ and the scale factor $b$ are known.

Calculating the limits $c->0$ and $c->1$ we can calculate the corresponding expressions for the $B S$ and Hill-Sips isotherms.

In the first case when $c->0$ and using the properties of the function Gamma in Equation (64), one has for any positive value $a$.

$$
\begin{aligned}
& \langle\varkappa\rangle_{B S}=b \frac{1}{a} \Gamma\left(\frac{1}{a}\right) \\
& \left\langle\varkappa^{k}\right\rangle_{B S}=b^{k} \Gamma\left(\frac{a+k}{a}\right) \\
& b^{a}=\left\langle\varkappa^{a}\right\rangle_{B S} \quad a>0
\end{aligned}
$$

For the second case one gets

$$
\begin{aligned}
& \langle\varkappa\rangle_{H S}=b \frac{\pi}{a} \operatorname{Csc}\left(\frac{\pi}{a}\right) \quad a>1 \\
& \left\langle\varkappa^{k}\right\rangle_{H S}=b^{k} \frac{\pi k}{a} \operatorname{Csc}\left(\frac{\pi k}{a}\right) \quad a>k
\end{aligned}
$$

It is convenient to have a relation between $a$ and $b$ valid for all positive values of $a$. This can be obtained using (64) and the properties of the Gamma function:

$$
b^{a}=\frac{4}{\pi^{2}}\left\langle\varkappa^{\frac{a}{2}}\right\rangle_{H S}^{2} \quad a>0 .
$$

More results can be found in [52].

All these results can be obtained directly by performing the corresponding integrals.

The characterization of sorption using the values of $\Theta_{\max }, a, b$, and $c$ obtained by the best fit of experimental isotherms using the new methodology derived in this paper is in preparation. 
Scientific Research Publishing (SCIRP) is one of the largest Open Access journal publishers. It is currently publishing more than 200 open access, online, peer-reviewed journals covering a wide range of academic disciplines. SCIRP serves the worldwide academic communities and contributes to the progress and application of science with its publication.

Other selected journals from SCIRP are listed as below. Submit your manuscript to us via either submit@scirp.org or Online Submission Portal.
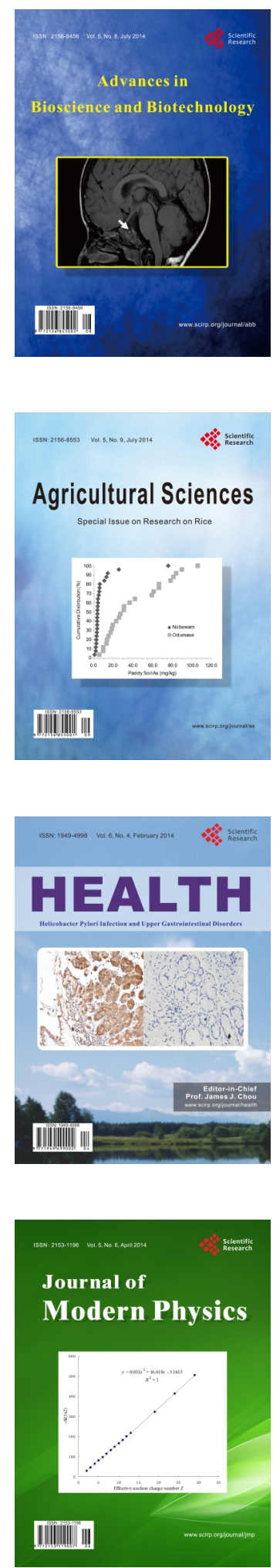
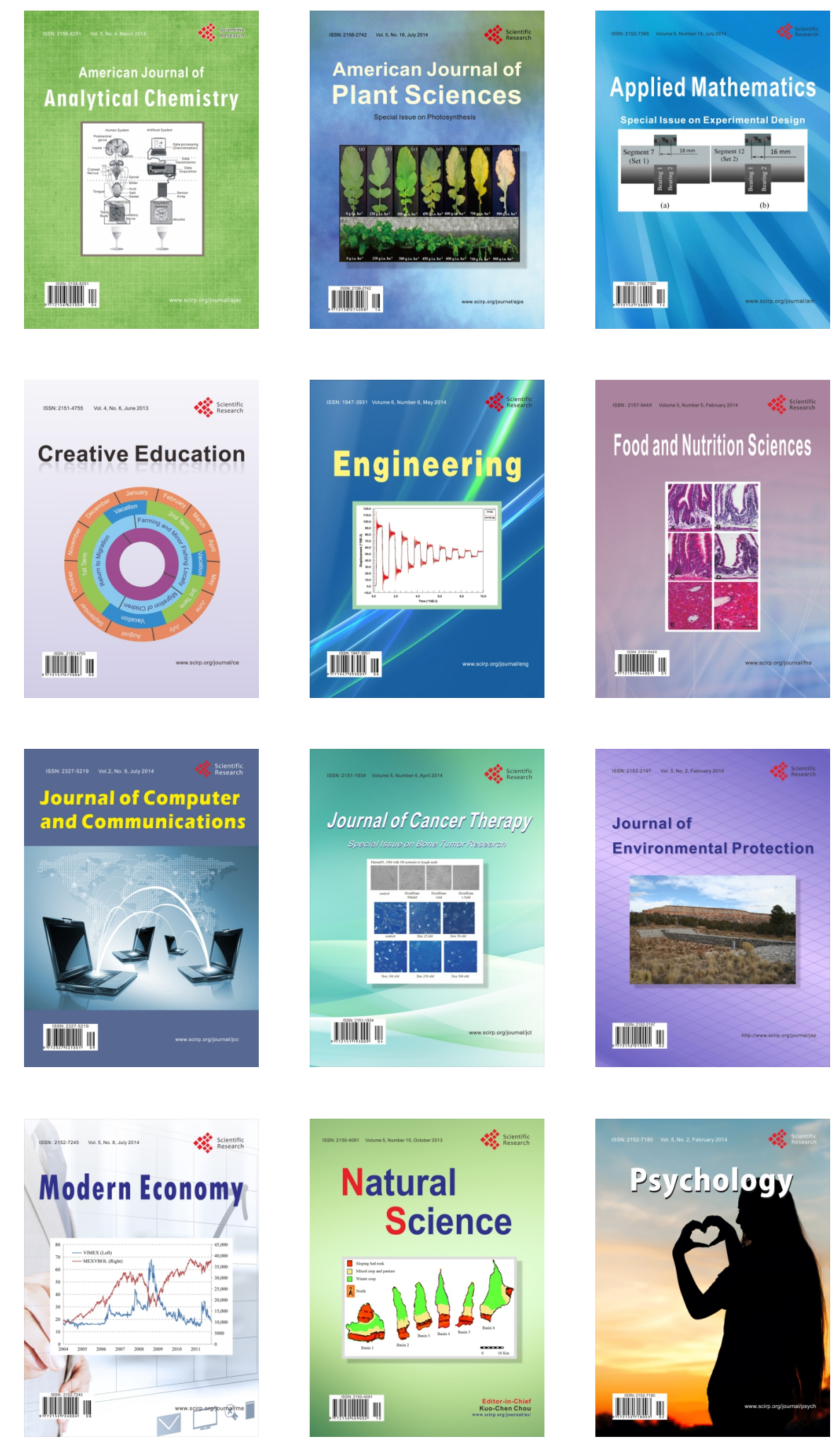